\title{
Characteristics of variable speed limit systems
}

\author{
Ellen F. Grumert ${ }^{1,2^{*}}$ (D), Andreas Tapani ${ }^{1}$ and Xiaoliang $\mathrm{Ma}^{3}$
}

\begin{abstract}
The control algorithm used for deciding on the speed limit in variable speed limit systems is crucial for the performance of the systems. The algorithm is designed to fulfil the purpose of the variable speed limit system, which can be one or several of the following aspects: increasing safety, increasing efficiency and decreasing environmental impacts. Today, many of the control algorithms used in practice are based on fixed thresholds in speed and/or flow. Therefore, they are not necessarily reflecting the current traffic conditions. Control algorithms with a greater level of complexity can be found in the literature. In this paper, four existing control algorithms are investigated to conclude on important characteristics affecting the performance of the variable speed limit system. The purpose of the variable speed limit system and, consequently, the design of the control algorithm differ. Requirements of the investigated control algorithms are that they should be easy to interpret and the execution time should be short. The algorithms are evaluated through microscopic traffic simulation. Performance indicators related to traffic safety, traffic efficiency and environmental impacts are presented. The results show that the characteristics of the variable speed limit system and the design of the control algorithm will have effect on the resulting traffic performance, given that the drivers comply with the variable speed limits. Moreover, the time needed to trigger the system, the duration and the size of speed limit reductions, and the location of the congestion are factors of importance for the performance of variable speed limit systems.
\end{abstract}

Keywords: Variable speed limit systems, Control algorithms, Microscopic traffic simulation

\section{Introduction}

Since its beginning, road traffic has increased tremendously leading to congestion, safety problems and environmental issues $[20,21]$. Today, urban motorways around the world are experiencing daily congestion during peak-hours. Hence, there is a need for traffic management aiming at reducing the congestion and thereby increasing both efficiency and safety. One commonly applied traffic management system for urban motorways is variable speed limit (VSL) systems. VSL systems make use of a control algorithm to adjust the speed limit based on the prevailing road and traffic conditions. Coordinated variable speed limits are shown on a series of variable message signs along the managed road. Information about traffic

*Correspondence: ellen.grumert@vti.se

'Swedish National Road and Transport Research Institute (VTI), SE-581 95 Linköping, Sweden

${ }^{2}$ Linköping University, Department of Science and Technology, SE-601 74 Norrköping, Sweden

Full list of author information is available at the end of the article conditions is collected using detectors often measuring local speed, flow or detector occupancy. A suitable variable speed limit is calculated by the control algorithm taking into account downstream and upstream traffic conditions. The purpose of a variable speed limit system can be to increase safety, increase efficiency and/or decrease environmental impacts, and hence, the specific purpose is reflected in how the control algorithm is designed.

When implementing a VSL system the purpose of the system but also the complexity and the level of detail of the control algorithm are of importance. It is desirable to have a control algorithm that is reflecting the specific purpose of the system. However, at the same time it is desirable to have a control algorithm that is straightforward to implement. For example, the required control input should be easy to measure, the current traffic conditions should be reflected in the speed limit change and the tuning parameters should be easy to interpret. Additionally, the control algorithm should be able to run in real-time without computational delay. This results in a trade-off between the

\section{Springer Open}

(c) The Author(s). 2018 Open Access This article is distributed under the terms of the Creative Commons Attribution 4.0 International License (http://creativecommons.org/licenses/by/4.0/), which permits unrestricted use, distribution, and reproduction in any medium, provided you give appropriate credit to the original author(s) and the source, provide a link to the Creative Commons license, and indicate if changes were made. 
complexity of the algorithm and the degree of which the algorithm is able to fulfil its defined purpose. VSL control algorithms used in practice are often rule-based, only using a threshold of measured speed or flow as a basis for lowering the speed limit. More elaborate control algorithms are proposed in the literature, but there are few studies comparing how the characteristics of these algorithms relates to each other and the final outcome of the systems.

In this paper, we aim to investigate how the design of the control algorithm and the defined purpose of the VSL system affect the performance of the traffic system. Further, the objective of the study is to summarize characteristics that should be considered when designing a VSL system. Consequently, this study contributes to knowledge on the relationship between traffic conditions and variable speed limits.

We investigate four existing VSL control algorithms; two of them proposed in the literature, one proposed in the literature and implemented as a field trial and one implemented in real traffic. The algorithms differ by their objective and thereby also by the aspects included in the control algorithm. Two of the control algorithms are rule-based, but with different update rules [30, 52], one algorithm is control-theory based [4] and one algorithm is based on analytical expressions [18]. The algorithms are evaluated, with respect to traffic efficiency, safety and environmental impact, by the use of microscopic traffic simulation. This paper is the result of further analysis and extension of a previous comparison of VSL control algorithms [13].

The remainder of the paper is organized as follows. First, an introduction is given to variable speed limit systems and to the four VSL control algorithms. This is followed by a presentation of the evaluation method using microscopic traffic simulation and a description of the simulation scenario. Then, simulation results are presented and discussed. Finally, conclusions from the study are given.

\section{Variable speed limit systems}

Variable Speed Limit (VSL) systems consist of a series of VSL signs and associated detectors measuring the traffic conditions. The speed limits shown on the VSL signs are based on the observed traffic conditions close to the VSL sign, but usually also the traffic conditions at upstream and downstream VSL signs. Independent variable speed limits were first introduced in the 1960's [36] and as a system for motorway traffic control in the 1970's [46]. Two main approaches exist $[23,36,46]$ and have been differently defined in different studies. These two main approaches are in this study defined as:

1. Incident detection systems: increasing safety by reducing the speed limit substantially when an incident has occurred and thereby decreasing the probability of further incidents and

2. Homogenization systems: preventing a traffic breakdown by applying a slightly reduced speed limit when the traffic flow is close to capacity to avoid unstable traffic conditions.

In recently proposed systems, the goal has also been to reduce environmental impacts and to relocate flow to other parts of the network to reduce exhaust emissions, see e.g. (Gao, K: Multi-objective traffic management for livability, Improve Near-motorway Livability in the Netherlands. MS. Thesis, unpublished) [57].

The first applications of VSL systems were incident detection systems with the purpose to warn the drivers of the incident and to decrease the risk of additional incidents. Examples of implemented incident detection systems are the ones included in the Swedish [52] and the Dutch [50] Motorway Control System (MCS). A combination of incident detection and homogenization are included in some VSL systems, such as in the VSL system in the UK [37]. Studies from implemented systems show benefits in traffic safety and flow homogenization (reduction of the variance in speed between vehicles), while improvements in traffic efficiency are limited. As a result of increased costs of delays due to congestion, traffic efficiency has also become an important aspect to consider in the design of a VSL system. It is well-known that the capacity at the onset of congestion tend to drop, leading to an inefficient traffic system. The drop has been discovered in many empirical studies, see e.g. [7, 48, 59]. In [59] and [27] the capacity drop is partly explained by an increased number of lane-changes during unstable traffic conditions. Another effect of lane-changing operations is stop-and-go traffic, as shown in [1] and [9]. For this reason, homogenization VSL systems focusing on reducing differences in speed amongst the vehicles, and thereby also reduce the demand for lane-changes have been proposed in the literature. By reducing the speed limit at the onset of congestion, the capacity drop and flow breakdown, can be delayed or even avoided. The systems are therefore not necessarily triggered when the mean speed on the road becomes low. Soriguera et. al. [46] has concluded that empirical studies, as early as 1972, indicate that homogenization can be obtained with an appropriate speed limit when the flow is close to the capacity. Also, many of the studies, including [46], show that a higher critical density can be obtained without notably reducing the traffic flow. Hence, by applying homogenization systems, traffic throughput can be increased, which will lead to increased traffic efficiency.

Four different types of VSL control algorithms are commonly proposed in the literature: rule based, fuzzy-logic based, analytical and control-theory based. The design 
of the control algorithms are reflected in the purpose of the VSL system and the required level of detail in the implementation. The simplest VSL systems include rulebased VSL control algorithms. The algorithms use thresholds for identifying incidents and situations with low speeds. Most of the VSL systems used in practice are rulebased. Examples of implemented rule-based VSL control algorithms are included in the UK [37], the Swedish [52] and the Dutch [50] Motor way Control Systems (MCS) and the Spanish dynamic speed limit system [47]. Examples in the literature are given in [2,30,32]. Another type of VSL control algorithm is the fuzzy-logic based VSL control algorithms, where the speed limit is decided based on how well the measured input matches a set of rules, see e.g. $[6,31,34]$. In analytical VSL control algorithms the purpose is to analytically calculate the traffic states based on a measured reality, see e.g. $[14,18]$. Finally, control theory based VSL control algorithms have been proposed to find the optimal VSL strategy based on local feedback loops, such as the algorithms proposed in [4] and [22] or by using model predictive control, see e.g. $[10,15,16,58]$.

\section{Variable speed limit algorithms in this study}

Four existing VSL control algorithms are investigated; two of them proposed in the literature, one proposed in the literature and implemented as a field trial and one implemented in real traffic. Requirements imposed on the control algorithms are that they should be straightforward to implement and easy to interpret. The execution time should be short and at the same time the applied variable speed limits should correspond to the purpose of the VSL system. Two of the control algorithms have as objective to increase safety, while two of them have as objective to increase traffic efficiency.

The first algorithm of this study is the rule-based incident detection algorithm used in the Swedish Motorway Control System (MCS) [52]. From here on it is referred to as MCS. The second algorithm in the study, by us referred to as the Reducing Crash Potential (RCP) algorithm, is proposed by Lee et. al. [30]. The algorithm is a rule-based incident detection algorithm but has a somewhat different approach compared to the MCS since the main goal is to reduce the risk of incidents by considering a crash potential instead of the measured mean speed. The objective is to avoid dangerous situations by detecting the crash potential at known problematic locations with a high risk of an incident, and to reduce the crash potential by lowering the speed limit. The third algorithm of the study, the SPEed ControlIng ALgorithm using Shockwave Theory (SPECIALIST) presented by Hegyi et. al. [18], has as objective to reduce the effects of shockwaves by lowering the speed limit. The shockwaves are detected using measurements from stationary detectors. An algorithm including linear equations is applied to resolve identified shockwaves in order to increase throughput at the resulting bottleneck. Hence, it can be seen as a combination of an incident detection system and a homogenization system. By lowering the speed limit to a beforehand determined value, the relationship between flow and speed will change in accordance with traffic flow theory. As a result, effects of shockwaves moving upstream can be reduced and, hence, traffic efficiency can be improved. Finally, the fourth algorithm of the study is the Motorway Traffic Flow Control (MTFC) algorithm [4, 5, 38, 39]. The objective of this algorithm is to maintain a stable traffic situation by keeping a detected occupancy as close to the capacity level as possible, and thereby avoiding a capacity drop. Since the speed limit is lowered with the objective to avoid unstable traffic conditions it is categorized as a homogenization algorithm. The algorithm is applied to known bottlenecks, where congested situations are frequently observed.

The Swedish MCS has been evaluated through empirical studies $[41,42]$. These studies concluded that the main effect of the MCS is increased safety. This is in line with a study comparing the Swedish and the Dutch MCS [27]. However, in the Netherlands accidents decreased by $20 \%$ but in Sweden only homogenized speeds and lower speed levels were found. This can be explained by the fact that the variable speed limits are not compulsory in Sweden. Further, the journey times was reduced by $10-15 \%$ in the Netherlands, compared to in Sweden where no such effect was observed. This is most probably the result of fewer accidents that resulted in a reduction in travel time. The RCP algorithm has been evaluated through microscopic traffic simulation $[29,30,56]$. The results presented in $[29,30]$ show a trade-off between safety, measured as decreased crash potential and traffic efficiency, measured as total time spent. Also, it is concluded in [30] and [56] that there are threshold values for the crash potential for which benefits in terms of both safety and efficiency can be made. In a field trial of SPECIALIST [17] it is shown that the algorithm is able to resolve around $80 \%$ of the identified shockwaves with a gain in travel time as a result. Hegyi et. al. [18] use macroscopic traffic simulation to conclude that SPECIALIST gives a decrease in total travel time spent and on average an increase in throughput of traffic flow. The MTFC has been evaluated using both macroscopic traffic simulation $[4,5]$ and microscopic traffic simulation $[38,39]$. The studies show improvements in efficiency, with reductions as high as $40 \%$ in total travel time. The details of the algorithms and the parameter settings used in this study are presented below.

\subsection{The MCS algorithm}

The VSL algorithm included in the MCS [52] uses thresholds for lowering and increasing the speed limit. One detector and one VSL sign is used for each lane but a common speed limit is applied for all lanes. The algorithm 
used for deciding on speed limit, $v_{t, j}$, at time $t$ and detector location $j$ are based on the detector measurements, $\tilde{v}_{t, j}$, and the use of the following speed limit updating scheme:

- If $\tilde{v}_{t, j} \leq 45 \mathrm{~km} / \mathrm{h}:$

- The speed limit at detector station $j$ is set to $v_{t, j}=60 \mathrm{~km} / \mathrm{h}$.

- Lead-in speed limits at two upstream detector stations are set to $v_{t, j-1}=80 \mathrm{~km} / \mathrm{h}$ and $v_{t, j-2}=100 \mathrm{~km} / \mathrm{h}$, respectively.

- If $\tilde{v}_{t, j} \geq 45 \mathrm{~km} / \mathrm{h}:$

- The speed limit at detector station $j, v_{t, j}$, and the associated lead in speed limits, $v_{t, j-1}$ and $v_{t, j-2}$, are reset to $120 \mathrm{~km} / \mathrm{h}$.

The thresholds are based on a study of the existing MCS and the new speed limits that are recently implemented in Sweden [35]. It is assumed that the most restrictive lane is regulating the speed limit, i.e. the lane with the lowest mean speed is considered when determining a common speed limit for all lanes. The algorithm is repeated at all detectors starting from the last downstream detector.

\subsection{The RCP algorithm}

In the RCP algorithm [30] VSLs are applied to a beforehand known bottleneck and calculated based on the current value of the crash potential at the bottleneck. The crash potential, $F_{t, j}$, for detector station $j$ at time $t$ is calculated by using a regression model estimated based on accident and traffic data.

$$
F_{t, j}=e^{\hat{\Theta}+\lambda_{C V S_{t, j}}+\lambda_{Q_{t, j}}+\lambda_{C O V V_{t, j}}+\lambda_{R_{t, j}}+\lambda_{P_{t, j}},}
$$

where $\hat{\Theta}$ is a constant. The crash precursors used in the model are the spatial and temporal variation in speed, $\lambda_{Q_{t, j}}$ and $\lambda_{C V S_{t, j}}$, the covariation in traffic volume between upstream and downstream locations, $\lambda_{C O V V_{t, j}}$, and external factors, $\lambda_{R_{t, j}}$ and $\lambda_{P_{t, j}}$. The calculations of crash precursors are described in [28-30]. VSL activation is determined based on threshold values of the crash potential. The risk of a crash is assumed to be highest just before the bottleneck. Therefore, the VSL is applied to a road stretch of 1000 meter just upstream of the bottleneck. Lead-in speed limit is applied on one section upstream of the highrisk section. We use the threshold value for lowering the speed limit given in [30]. Both the VSL and the lead-in speed limit are based on measurements of speed to reflect the conditions on the road, and are rounded to the nearest $10 \mathrm{~km} / \mathrm{h}$. The variable speed limit and the lead-in speed limit is calculated as

$$
v_{j}=\frac{\left(\tilde{v}_{j-1}+\tilde{v}_{j+1}\right)}{2},
$$

where $\tilde{v}_{j}$ is the mean speed measured at detector station $j$. The intervention time, i.e. the time when the VSL system is activated, is set to $10 \mathrm{~min}$. The set of parameters used in the simulation are based on the near-optimal set of parameters resulting from the calibration presented in [19] and [43].

\subsection{The SPECIALIST algorithm}

The SPECIALIST algorithm [18] uses shockwave identification to identify different states in speed and flow. The algorithm is developed to detect and resolve moving shockwaves. An extension also considering on-ramp flows are proposed in [44]. Linear equations are used to calculate the length and duration of the different states. Therefore, the length of the VSL activation area is depending on the level of congestion, the length of the congested area, etc. Also, the time of activation is based on the traffic conditions. Figure 1 gives an illustration of the algorithm.

The algorithm includes four phases related to the identified traffic states:

- Phase 1: The thresholds for identification of a shockwave and activation of the system are based on speed and flow. The system is activated when the mean speed and mean flow are below $50 \mathrm{~km} / \mathrm{h}$ and $1500 \mathrm{veh} /$ hour/lane, respectively. This results in a start and end point of the congested state 2 .

- Phase 2: A predefined lower speed limit is set to 60 $\mathrm{km} / \mathrm{h}$ and applied to state 2 and an additional stretch upstream of the congested state, state 3 . Additionally, state 4 becomes present as a result of vehicles starting to slow down before entering to state 3 . The tail of state 4 will propagate upstream or downstream depending on the flow levels in the state. The predefined speed limits are applied along with the propagation of the tail. Lead-in speed limits at two upstream detectors when the system is activated are 80 and $100 \mathrm{~km} / \mathrm{h}$, respectively. At the same time, speed limits are turned off downstream as the congestion is dissolved and state 3 propagates upstream.

- Phase 3: VSLs are applied to the tail of state 4 which is propagating upstream. At the same time, VLSs are turned off at the head of state 4 which is also propagating upstream. Downstream of state 4 there is a discharging area, state 5 , where the vehicles accelerate from speed levels corresponding to the speed limit in state 4 towards free flow speed.

- Phase 4: Eventually only state 5 remains together with the free flow states. No speed limit is applied. State 5 is propagating downstream until speeds corresponding to the maximum speed limit on the road are obtained. The predetermined parameters, as well as the presented thresholds, are set according to parameter settings 2 in [17]. 


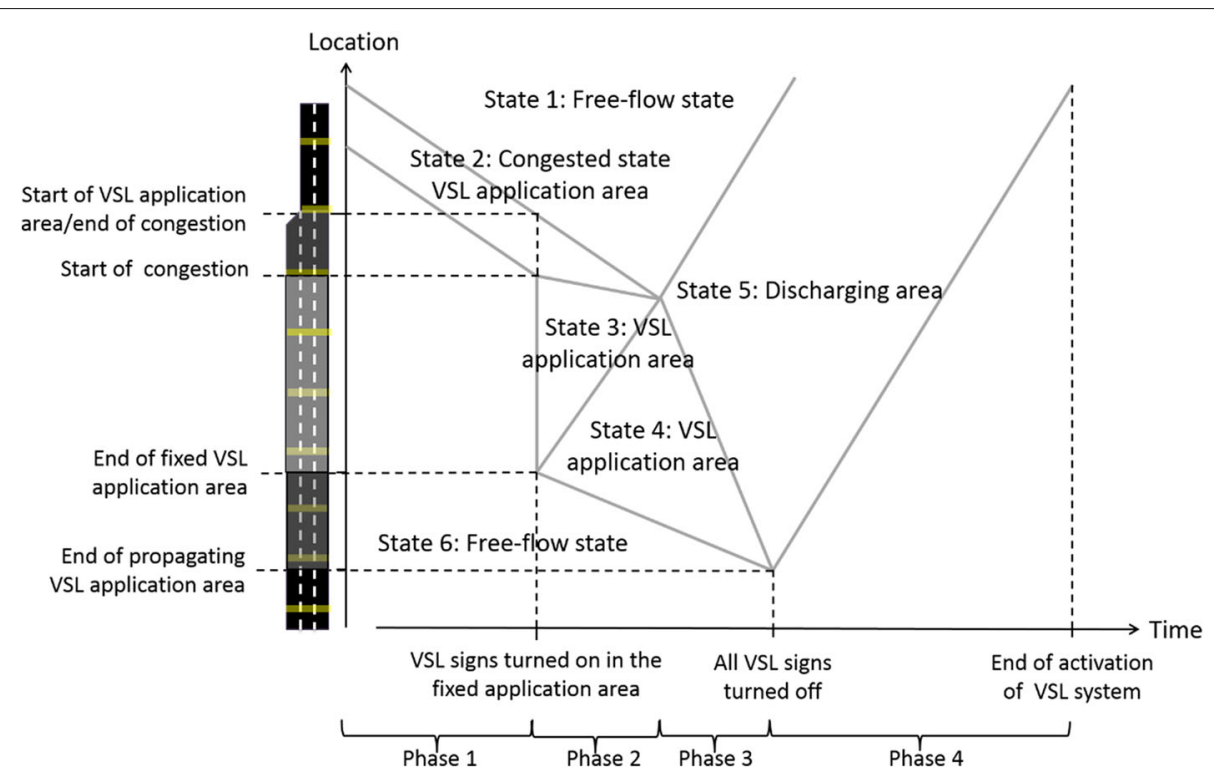

Fig. 1 Illustration of the different states and phases in the SPECIALIST algorithm

\subsection{The MTFC algorithm}

In the MTFC algorithm [39] the speed limit is determined by controlling the occupancy at an identifiable bottleneck towards a predefined estimate of the critical occupancy at the specific bottleneck. The variable speed limit at time $\mathrm{t}$ is determined as a fraction, $b(t)$, of the original speed limit on the road.

$$
b(t)=b(t-1)+K_{I}^{\prime} e_{o}(t),
$$

where $K_{I}^{\prime}$ is the integral gain and $e_{o}(t)$ is the occupancy error calculated as the difference between the critical occupancy, $\hat{o}_{\text {out }}$, and the measured occupancy at the bottleneck, $\tilde{o}_{\text {out }}(t)$. The occupancy at capacity flow is in this study set to $13 \%$ based on an investigation [12] of the capacity level in the base case scenario in SUMO. The threshold is set to $1 \%$ below the critical level of the occupancy to limit the risk of exceeding the capacity. Algorithm specific parameters are taken from the study presented in [39]. The VSLs varies between 20 and 100\% of the original speed limit on the road and are rounded to the nearest $10 \mathrm{~km} / \mathrm{h}$. Four detectors located around the bottleneck are included in the system. The occupancy measurement used in the algorithm is the maximum of the occupancy measurements of these four detectors. The speed limit is applied to an application area of 300 meter, located 275 meters upstream of the bottleneck.

\section{Evaluation method}

We analyze the traffic performance resulting from the use of the four algorithms in a variable speed limit system and a base case without VSLs. This is done in order to investigate how the level of detail of the algorithm and the purpose of the VSL system are affected by the type of control algorithm. The base case and the algorithms are implemented and evaluated using the microscopic traffic simulator SUMO [8, 25]. Performance indicators related to traffic efficiency, safety and environmental impact are considered.

\subsection{Modeling VSL control algorithms in SUMO}

For this study microscopic traffic simulation is a suitable method as it is necessary to keep track of individual vehicles' reactions toward changes in VSLs. SUMO is open source, multi-modal, space continuous and time discrete. The car-following model used to model vehicle interactions is developed by Krauß [26] and based on the calculation of a safe speed, cf. the approach of Gipps [11]. The lane changing model is rule based. The core models in SUMO is further described by Krajzewicz [24]. The VSL algorithms are implemented through python scripts [51]. SUMO's Traffic Control Interface (TraCI) is used for communication of the speed limits to be applied on each road segment, and for accessing the mean values gathered by the detectors in the simulation.

When a speed limit is updated, the vehicles in the simulation receive and adapt to this information according to the modeling of vehicle movements set out by SUMO. The speed limit is adjusted based on the specific algorithm, and an updated speed limit is assigned to a road segment in the simulation when needed.

As a result, all vehicles on a road segment are assumed to get information about the new speed limit irrespective of their location. An alternative would be to assume that the vehicles get information about a speed limit 
change only when passing a variable speed limit sign. The assumption made is a simplification and is the same for all the algorithms included in the comparison. As a result of the simplification the vehicles in the simulation are adapting to a decrease, or an increase, in speed limit slightly earlier in time than what would be the case in reality. As indicated in an earlier microscopic traffic simulation based study [39], this will result in a more effective VSL system and smoother transitions towards the new speed limits compared to if information about a speed limit change only becomes available at specific points in space. Further, the authors conclude that the smoother transitions will result in that no temporary drop in capacity and flow will appear at the bottleneck at the activation of the VSL system. Also, for this reason, the difference in speed between two segments becomes larger when the VSL system is active.

The number of detectors and VSL signs are varying depending on the applied algorithm. In MCS and SPECIALIST, the study includes fourteen equipped segments located 500 meters apart from each other, and with one detector and one VSL sign applied to each lane. For RCP, the bottleneck location is assumed to be located where the number of lanes change from three lanes to two lanes. Hence, the detectors and the VSL signs are applied to two 500-meter segments before the bottleneck. For MTFC the detectors are concentrated around the bottleneck and the VSL signs are applied just upstream of the bottleneck according to the description in Section 3. The time when new speed limits are updated and applied based on prevailing traffic conditions are the same irrespective of algorithm. The update time is set to $30 \mathrm{~s}$.

\subsection{Base case - simulation scenario}

In order to make a consistent comparison, the same base case is used as a starting point for the four algorithms. The base case consists of a three-lane road with a lane-drop bottleneck at which the number of lanes is decreased to two. By increasing the flow to the capacity of two lanes we model an incident in the form of bottleneck activation. The reason for choosing this simulation scenario as a base case is that it has been shown to give representative characteristics of an incident in SUMO [12]. Further, the simple scenario makes it possible to isolate the effects of the algorithms under identical traffic conditions. The simulated road included in the evaluation is divided into fourteen 500-meter segments. Further, a start and end segment are included to avoid boundary effects, resulting in a $9 \mathrm{~km}$ long simulated road. The bottleneck is located $7.5 \mathrm{~km}$ downstream of the start segment. The maximum allowed speed limit on the road is assumed to be 120 $\mathrm{km} / \mathrm{h}$. Figure 2 gives an illustration of the simulated road stretch.

The simulation is performed for a 55 -min period, excluding a warm-up period of 5 min to prevent from loading effects. First, the input flow is held constant at $1500 \mathrm{veh} / \mathrm{h}$ for $10 \mathrm{~min}$. Then, to activate the bottleneck and the algorithms, the flow is increased to $4500 \mathrm{veh} / \mathrm{h}$ for $15 \mathrm{~min}$, which is approximately $70 \%$ of the capacity of three lanes. Finally, the flow is decreased to $1500 \mathrm{veh} / \mathrm{h}$ for $30 \mathrm{~min}$ for the congestion to be resolved and the VSL signs to become inactive.

Mean values used in the algorithms for control of the VSLs are based on smoothed mean values of measurements over $30 \mathrm{~s}$ intervals. It is comparable to the means used in the design of the MCS algorithm [52]. The smoothed mean values consist of the previous mean and the measured mean, weighted equally with a smoothing factor of 0.5. The VSLs are assumed to be compulsory for the vehicles in the simulations. For a more detailed description of the implementation of the control algorithms see [12].

\subsection{Vehicle parameters}

Assumptions regarding the vehicle parameters are the same irrespectively of VSL system. The maximum acceleration ability is set to $0.8 \mathrm{~m} / \mathrm{s}^{2}$ and the reaction time is set to $1.3 \mathrm{~s}$ based on an investigation of merging behavior

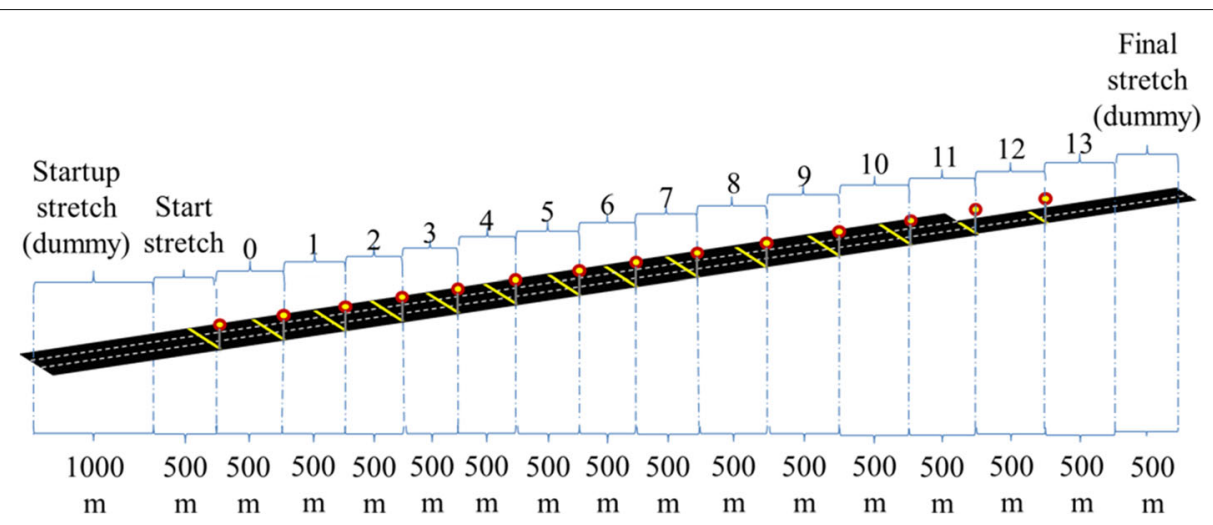

Fig. 2 Illustration of the simulated road stretch 
in SUMO [12]. The desired speed factors are drawn from a normal distribution with mean 1.0 and speed deviation 0.1, based on a study by Varedian [53] in which a speed distribution was estimated for all vehicle types on Swedish roads with a speed limit of $120 \mathrm{~km} / \mathrm{h}$. Thereby, we implicitly assume a typical composition of vehicle types in the simulated traffic. Differences in vehicle length are, however, not considered. Remaining vehicle parameters used in the simulation are set to default values used in SUMO version 0.19.0 [8].Vehicles are generated with exponentially distributed headways.

\subsection{Performance indicators}

The four algorithms included in the study are focused either on safety or efficiency. Hence, to evaluate how the objectives of the algorithms affect the results both safety and efficiency indicators have been included. None of the algorithms have as objective to reduce environmental impacts. Nonetheless, it is interesting to investigate how the algorithms perform in that respect. Therefore, a simple investigation of the environmental impacts is included.

The effectiveness of the different algorithms is measured by the mean speed over the simulated road. The mean speed is calculated as a rolling average over $30 \mathrm{~s}$ intervals for the whole road stretch to get the overall impact on traffic efficiency. The smoothing factor is 0.5 . A more detailed investigation of each of the algorithms is carried out by examining the mean speeds collected at the individual detector stations. The means and standard errors of the means are calculated based on 20 replications of the simulation for the base case and for each of the VSL systems considered.

The Coefficient of Variation of Speed (CVS) described in [30] is used as a measure of safety. The CVS is calculated for each lane $i$ and averaged over a segment $j$ as

$$
C V S_{j}=\frac{1}{n} \sum_{i=1}^{n} \frac{\sigma_{i}}{\bar{s}_{i}},
$$

where $\sigma_{i}$ and $\bar{s}_{i}$ is the standard deviation of speed and the mean speed, respectively. The $C V S_{j}$ is presented as a mean over 20 simulation runs.

Finally, the microscopic emission model CMEM [45] is used for evaluation of environmental impacts. The model gives second by second tailpipe emissions, $e_{\text {tailpipe }}$, as a product of three parameters, fuel rate $\left(r_{\text {fuel }}\right)$, engine-out emission index $\left(i_{\text {em/fuel }}\right)$ and catalyst pass fraction $\left(C_{\text {pass }}\right)$, that is

$$
e_{\text {tailpipe }}=r_{\text {fuel }} \cdot i_{\text {em } / \text { fuel }} \cdot C_{\text {pass }},
$$

In the evaluation, the vehicle specific parameters used in CMEM have been adopted for emission estimation based on the properties and the assumptions of the vehicles simulated in SUMO. Consequently, the vehicles are assumed to be of the normal emitting catalyst equipped gasoline fueled passenger car type in CMEM. Confidence intervals taking into account the 20 simulation runs are used to present the significance of the observed differences in emissions.

\section{Computational results}

In this section, results from the simulations are presented for all of the investigated algorithms and the base case. Results related to traffic efficiency, traffic safety and environmental impacts are given. This is followed by a discussion on how the different control algorithms manage to fulfil the specific purpose of the variable speed limit system and how the characteristics of each control algorithm are reflected in the results.

\subsection{Traffic efficiency}

Figure 3 shows the mean speed over the whole stretch. The base case without applying a VSL algorithm is plotted together with the four VSL systems. The standard error of the means presented is less than or equal to $4.14 \mathrm{~km} / \mathrm{h}$. From the figure, we conclude that only one algorithm, the MTFC, manages to increase the mean speed compared to the base case during congested conditions. Both the MCS and the RCP algorithms have mean speed levels that are slightly below the base case when the queue is building up and until the flow is decreasing again. The same holds for the SPECIALIST algorithm, except for the most congested period where the algorithm manage to keep a mean speed that is somewhat higher than the base case.

A more detailed analysis of the different algorithms is done by investigating the mean speed for each detector station, and for each VSL algorithm, as shown in Fig. 4.

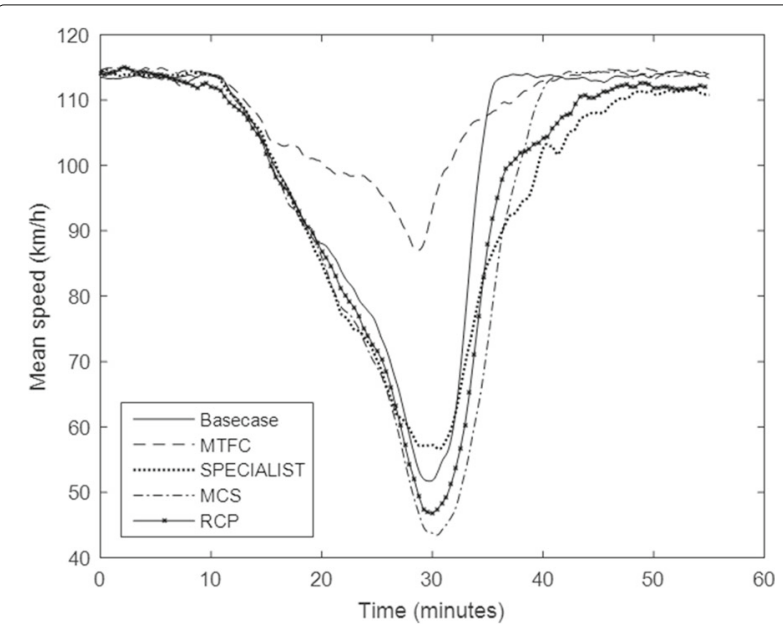

Fig. 3 Mean speed over the whole stretch for the base case and the VSL algorithms 

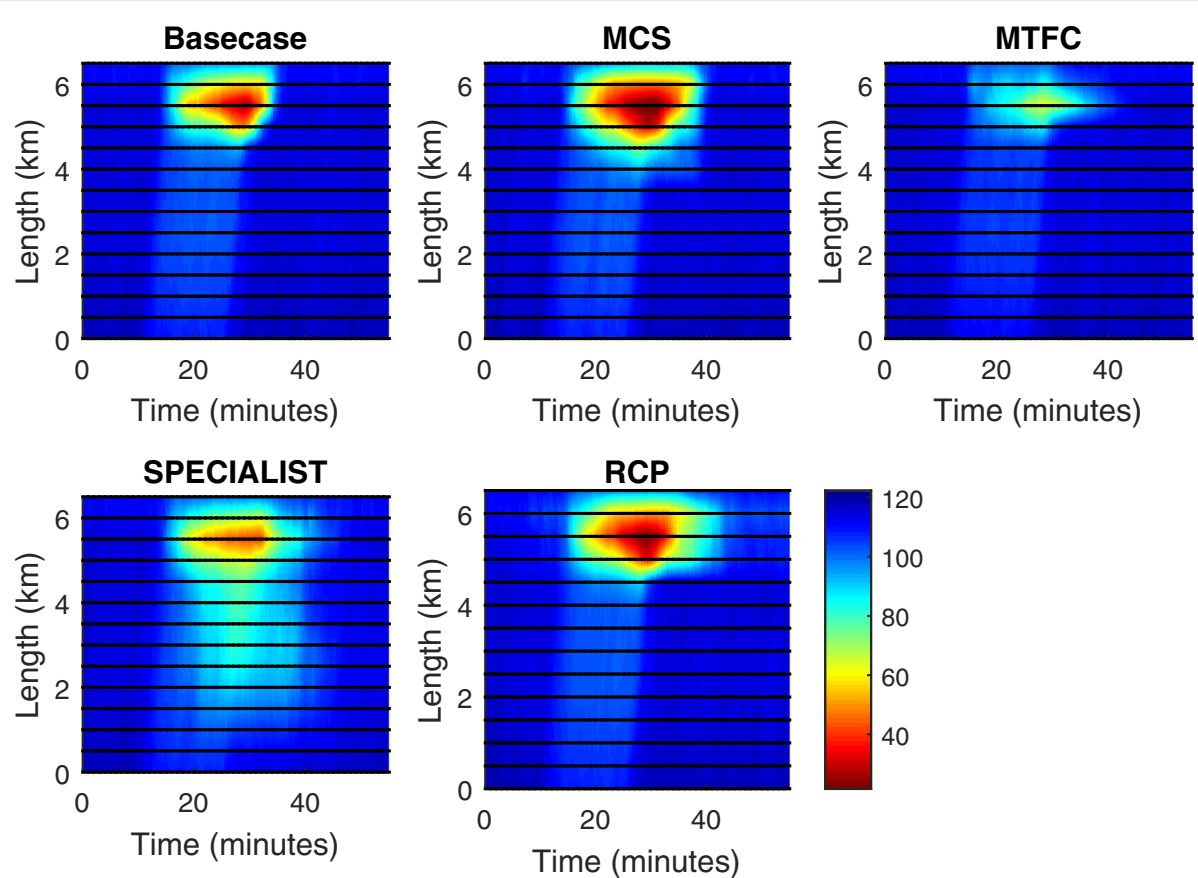

Fig. 4 Mean speed $(\mathrm{km} / \mathrm{h})$ at the detector stations for the base case and the VSL algorithms

In the figure, the mean speed ranges from 20 (dark red) to 120 (dark blue) $\mathrm{km} / \mathrm{h}$ and the horizontal lines represent the location of the detector stations. The standard error of means presented are less than or equal to $7.02 \mathrm{~km} / \mathrm{h}$.

As can be seen in Fig. 3 the MCS tends to have a mean speed slightly below the other algorithms under congested conditions. This is consistent with Fig. 4, where it is concluded that the mean speeds at all detectors are lower compared to the base case. Also, a larger area is influenced by lower mean speed compared to the base case.

For the MTFC substantially higher mean speed over the whole stretch, as well as for each detector station, are observed compared to the base case. In the congested period, the mean speeds are around $90 \mathrm{~km} / \mathrm{h}$ whereas in the base case the mean speeds ranges between $35 \mathrm{~km} / \mathrm{h}$ and $70 \mathrm{~km} / \mathrm{h}$ depending on detector station.

Traffic performance measured as mean speed over the whole stretch is lower for the SPECIALIST algorithm compared to the base case, except in the most congested period. This is explained by the results in Fig. 4. The mean speed is lower for most detectors, as a result of the lowering of speed limits upstream of the congestion. Nonetheless, close to the bottleneck, in the area between 4.5 and $6 \mathrm{~km}$, the mean speed is higher during the congested period. Further, the VSL application area is long. It is only at detector station 1 that the original speed limit on the road is kept for the whole simulation period.

As can be seen in Fig. 4 the RCP shows lower mean speeds than the base case at detector stations 10 and 11 (between 5 to $6 \mathrm{~km}$ ), where the speed limits are lowered during congested condition. But also, the mean speeds at detector stations close to the VSL controlled area are decreased compared to the base case.

\subsection{Traffic safety}

The CVS described in the Section Performance indicators is used as a measure of traffic safety. The CVS for each detector station is presented in Fig. 5. The CVS ranges from 0 (dark blue) to 0.3 (dark red). A high CVS corresponds to a large variation in speed, and thereby a decrease in safety. The standard error of means presented are less than or equal to 0.06 .

The CVS around the bottleneck are lower for the MCS and RCP compared to the base case. SPECIALIST shows a large area with high variation in speeds. Although for a small area at the bottleneck location, decreased levels of CVS are detected. Just after the bottleneck location much higher variations in speed are detected. Also at detector station 1 somewhat higher variations in speed are detected. The MTFC shows lower levels of CVS compared to the base case, and the other algorithms.

\subsection{Environmental impacts}

In Table 1 emission levels calculated using CMEM are presented. The environmental impacts measured with CMEM shows small differences when comparing the base case with the algorithms. MTFC gives the biggest improvements compared to the base case. Both the MCS and the RCP are performing somewhat worse than the base case. 

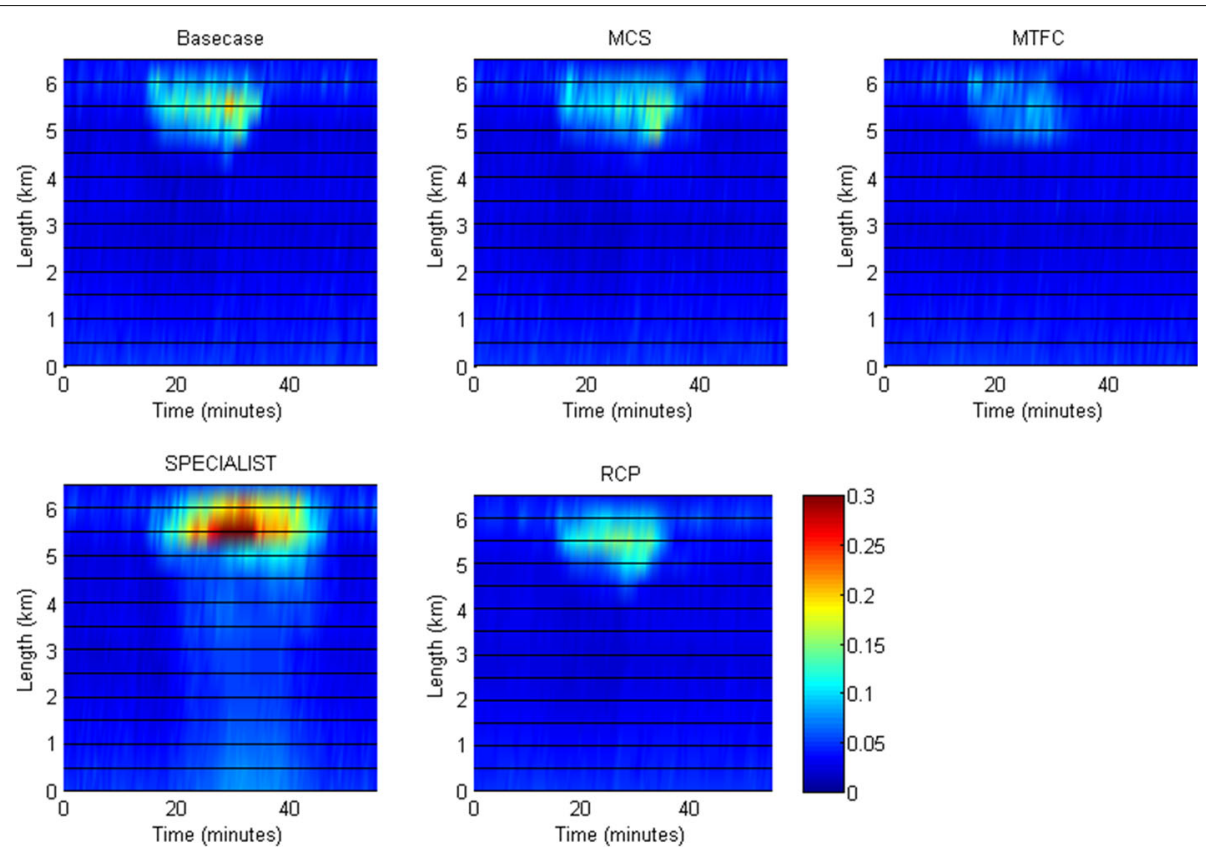

Fig. 5 The Coefficient of Variation of Speed (CVS) for the base case and the VSL algorithms

\section{Discussions}

The microscopic traffic simulation model has been calibrated for the capacity and speed distributions observed on Swedish roads. Consequently, the results presented indicate how the algorithms performs under typical Swedish conditions. However, the results should be valid for motorways with similar conditions regardless of country. By modeling congestion due to a lane drop, the effect of merging, which is usually hard to calibrate in microscopic traffic simulation, is limited. Thereby, the uncertainty related to limitations in the simulation model is reduced.

The first rule-based control algorithm, MCS, show increased performance in safety close to the bottleneck location due to the lowered speed limits and the leadin speed limits. However, the algorithm uses the most simplistic rules compared to the other algorithms, with a substantial lowering of the speed limit close to the congested area, which limit the improvements in traffic efficiency. The lead-in speed limits result in even larger reductions in efficiency by introducing additional delay in the traffic system. Further, the algorithm become active only when low speeds are detected and when a breakdown already occurred or is about to occur. The results from this study is in line with the objective of the algorithm and the findings from the literature. In an empirical study of the Swedish MCS no improvements were observed in traffic efficiency, but the variance in speed between vehicles were reduced leading to increased traffic safety [42]. Although, in [3] a decrease in travel time in the similar MCS in the
Netherlands is observed. This is most probably due to that the VSLs in the Netherlands are compulsory, while the VSLs in Sweden are only recommended. As a result, the Dutch VSL system manages to reduce travel times due to that the system is more effective in decreasing the number of additional incidents. In the studied scenario no larger incidents are simulated, and hence improvements due to avoidance of additional incidents cannot be observed.

The second rule-based control algorithm, RCP, does also show an increase in safety close to the bottleneck. Even so, the objective of reducing the crash potential by smoothing of the traffic flow is not reflected in the results, leading to that smaller effects on safety than expected are observed. This is probably a result of the local control with lower speed limits only on two of the road segments. As a result, the large differences in mean speed between segments is counteracting the objective of the algorithm. Hence, the objective of improving safety at the bottleneck is fulfilled but at the expense of decreased safety at other

Table 1 Environmental impacts for the base case and the VSL algorithms

\begin{tabular}{llll}
\hline VSL algorithm & Mean CO2 $(\mathrm{kg})$ & Mean HC $(\mathrm{g})$ & Mean NOx $(\mathrm{g})$ \\
\hline Base case & $1175 \pm 49$ & $1767 \pm 101$ & $3154 \pm 170$ \\
RCP & $1180 \pm 49$ & $1825 \pm 97$ & $3223 \pm 159$ \\
MCS & $1183 \pm 51$ & $1817 \pm 116$ & $3219 \pm 187$ \\
MTFC & $1172 \pm 45$ & $1671 \pm 85$ & $3023 \pm 143$ \\
SPECIALIST & $1165 \pm 47$ & $1754 \pm 104$ & $3124 \pm 169$ \\
\hline
\end{tabular}


locations along the road. In RCP, the focus is on safety, why a reduction in travel time is not necessarily observed. However, earlier studies of RCP $[29,30,56]$ showed a reduction in both the crash potential and in travel time. This is due to that the variable speed limits are reflecting the mean speed on the road, rather than a predefined much lower speed limit. In this study, based on manually adjusting the parameters, it was not possible to find a set of parameters which resulted in a reduction in both crash potential and an increase in mean speed and the resulting mean speeds are comparable to the base case. However, more extensive tuning of the parameters can improve the performance of the algorithm. Another explanation of the limited improvements in traffic efficiency are related to the recovery rate, i.e. the time when the algorithm is deactivated and traffic conditions return to free flow. RCP has predefined fixed VSL activation times that sometimes seem to be longer than necessary.

SPECIALIST can be seen both as an incident detection and a homogenization system. Since the system use thresholds to identify incidents (moving jams) it becomes active only when an incident has occurred. For SPECIALIST an increase in safety measured as CVS is in general not observed, with exception of some improvements at the bottleneck location. This can be explained by the long stretch with lowered speed limits, which result in less congestion and a higher mean speed around the bottleneck compared to the base case. As a consequence, the variations in speed are increasing in the area of the bottleneck. Even as far away as at the first detector station somewhat higher variations in speed are detected as a result of the starting point of the lowered speed limits being located there. However, the goal of preventing further incidents and homogenizing the traffic flow by the use of lead-in speed limits are reflected in the results. The longer areas with lowered speed limit are resulting in less abrupt speed transitions in space compared to the base case. Hence, safety is improved in the form of a more homogenous traffic flow. In earlier studies [17, 18], SPECIALIST gave decreased travel times. No overall improvements in travel time could be observed in this study. However, SPECIALIST managed to improve the traffic efficiency close to the bottleneck location. The lack of improvement in overall traffic efficiency for SPECIALIST can be explained by the fact that the control algorithm is designed for backwards propagating shockwaves. So even though the conditions for detecting a shockwave is fulfilled in the simulated scenario and an increase in speed is seen just at the bottleneck, the algorithm would probably work better for moving shockwaves. On the other hand, this means that the speed of the shockwave, and thereby the type of congestion, needs to be identified in order to avoid activation for other types of congestion such as accidents, incidents, etc. This is usually not possible and would require extensive detection equipment. Other explanations to the limited improvements in traffic efficiency are related to the time of activation, the recovery rate, the lead-in speed limits and the size of the speed limit reduction. Except from the size of the speed limit reduction, the factors are based on the estimated traffic states and the predefined parameters. Hence, one reason for the long VSL activation time and slow recovery rate, and the long application area, is the set of parameters applied in this study and, therefore, fine tuning of the parameters can improve the performance of the algorithm.

As expected MTFC performs best of the studied algorithms when it comes to improving traffic efficiency. This is a result of that the algorithm is working to prevent a potential breakdown before it actually occurs. Increased traffic efficiency for MTFC have also been observed in $[4,5,38,39]$. Additionally, traffic safety was increased as a result of the increased traffic efficiency. This is in line with a study where MTFC was compared to VSL algorithms with the objective to improve safety [33]. It should be noted that the algorithm works well at capacity levels and for short time periods. However, longer time periods and/or heavily congested conditions have not been investigated in this study. The system might give larger effects on traffic efficiency at higher flow levels, at least up to a certain level when the traffic becomes oversaturated.

None of the studied algorithms were found to have any substantial effect on emissions. Although, a more thorough investigation of the emissions might give more insight to how the algorithms may affect the environmental impact.

Finally, note that the MCS and the SPECIALIST can be applied for unknown bottleneck locations. Whilst, RCP and MTFC are applied at predefined bottleneck locations and with bottleneck specific parameters. Identification of the bottleneck location and the bottleneck specific parameters can be done based on offline calibration as in this paper, or based on online calibration as described in [54], [49] and [55].

\section{Conclusions}

In this study, we have investigated how the performance of VSL systems is affected by the design of the control algorithm and the purpose of the system. Four algorithms, existing in the literature and implemented in real traffic, with different objectives are investigated. The results show that all of the algorithms are activated close to capacity levels and the objective of all the algorithms are fulfilled close to the bottleneck. It is concluded that the design of the control algorithm and the purpose of the VSL system will have an impact on which aspects of the traffic conditions that are affected by the system. Desirable characteristics when implementing a control algorithm are fast reactions to changes in the traffic conditions, early 
incident detection, limited duration and application area of the variable speed limit. These desirable characteristics are independent of the purpose of the VSL system. All of the control algorithms have different predefined parameters that are related to the site specific traffic conditions. These parameters have to be carefully considered and tuned to increase the resulting traffic performance. No effects were observed with respect to environmental impacts. However, this is not the main purpose of any of the studied systems. Further, it is concluded that if the goal is to increase safety, there are no or only limited positive effects on traffic efficiency. But, if the goal is to increase efficiency, there can also be positive effects on safety.

Only algorithms using the current and historical traffic state as input have been investigated and compared. Many predictive algorithms also exists in the literature $[16,40$, 57]. A similar study including predictive algorithms will also be of interest. Moreover, further sensitivity analyses of each algorithm will increase the understanding of the behavior of the algorithms. Other relevant directions for future studies include; a study of a combination of an incident detection system and a homogenization system and incorporation of connected vehicle systems such as vehicle-to-vehicle and vehicle-to-infrastructure communication for incident and congestion detection. Also, considering a real use case based on site specific data will indicate how the VSL systems performs under more complex scenarios. The findings in this study are of use for both design and implementation of future VSL systems and for improvements of already existing VSL systems.

\section{Acknowledgement}

The authors would like to thank the Swedish Transportation Administration (Trafikverket) for financial support.

\section{Publisher's Note}

Springer Nature remains neutral with regard to jurisdictional claims in published maps and institutional affiliations.

\section{Author details \\ ${ }^{1}$ Swedish National Road and Transport Research Institute (VTI), SE-581 95 Linköping, Sweden. ${ }^{2}$ Linköping University, Department of Science and Technology, SE-601 74 Norrköping, Sweden. ${ }^{3}$ KTH Royal Institute of Technology, Department of Civil and Architecture Engineering, Teknikringen 10, SE-100 44 Stockholm, Sweden.}

Received: 14 November 2017 Accepted: 5 April 2018

Published online: 31 May 2018

\section{References}

1. Ahn S, Cassidy MJ (2007) Freeway traffic oscillations and vehicle lane-change maneuvers. In: Allsop RE, Bell MGH, Heydecker BG (eds). Transportation and Traffic Theory 2007. Papers Selected for Presentation at ISTT17, a peer reviewed series since 1959. Elsevier, London. pp 690-710

2. Allaby P, Hellinga B, Bullock M (2007) Variable speed limits: Safety and operational impacts of a candidate control strategy for freeway applications. IEEE Trans Intell Transp Syst 8(4):671-680

3. Berg S, Bukkems N (2001) Dutch and swedish experiences of the dutch motorway control system $\mathrm{mtm}$. In: Proceedings of the $8^{\text {th }}$ World
Congress on Intelligent Transport Systems. ITS America, ITS Australia, ERTICO (Intelligent Transport Systems and Services-Europe), Sydney

4. Carlson R, Papamichail I, Papageorgiou M (2011) Local feedback-based mainstream traffic flow control on motorways using variable speed limits. IEEE Trans Intell Transp Syst 12(4):1261-1276

5. Carlson RC, Papamichail I, Papageorgiou M (2013) Comparison of local feedback controllers for the mainstream traffic flow on freeways using variable speed limits. J Intell Transp Syst 17(4):268-281

6. Chiou YC, Huang YF, Lin PC (2012) Optimal variable speed-limit control under abnormal traffic conditions. J Chin Inst Eng 35(3):299-308

7. Chung K, Rudjanakanoknad J, Cassidy MJ (2007) Relation between traffic density and capacity drop at three freeway bottlenecks. Transp Res B Methodol 41:82-95

8. DLR and contributors: Sumo homepage (2013). Avaliable athttp://sumo. dlr.de/wiki/Main_Page

9. Duret A, Ahn S, Buisson C (2009) Spatio-temporal analysis of impacts of lane changing consistent with wave propagation. In: Transportation Research Board $87^{\text {th }}$ Annual Meeting, Compendium of papers, Washington, D.C. pp 16-p

10. Frejo JRD, Núñez A, De Schutter B, Camacho EF (2014) Hybrid model predictive control for freeway traffic using discrete speed limit signals. Transp Res C Emerg Technol 46:309-325

11. Gipps P (1981) A behavioural car-following model for computer simulation. Transp Res B Methodol 15(2):105-111

12. Grumert E (2014) Cooperative variable speed limit systems : Modeling and evaluation using microscopic traffic simulation. Licentiate thesis, Linköping University. Linköping University Press, Sweden. No. 1670

13. Grumert E, Tapani A (2016) Evaluation of four control algorithms used in variable speed limit systems. In: Transportation Research Board 95 ${ }^{\text {th }}$ annual meeting, Compendium of papers. Transportation Research Board, Washington, D.C

14. Han Y, Chen D, Ahn S (2017) Variable speed limit control at fixed freeway bottlenecks using connected vehicles. Transp Res B Methodol 98: $113-134$

15. Han Y, Hegyi A, Yuan Y, Hoogendoorn S, Papageorgiou M, Roncoli C (2017) Resolving freeway jam waves by discrete first-order model-based predictive control of variable speed limits. Transp Res C Emerg Technol 77:405-420

16. Hegyi A, De Schutter B, Hellendoorn J (2005) Optimal coordination of variable speed limits to suppress shock waves. IEEE Trans Intell Transp Syst 6(1):102-112

17. Hegyi A, Hoogendoorn S (2010) Dynamic speed limit control to resolve shock waves on freeways - field test results of the specialist algorithm. In: IEEE Conference on Intelligent Transportation Systems, Proceedings, ITSC'10. IEEE, Madeira Island. pp 519-524

18. Hegyi A, Hoogendoorn S, Schreuder M, Stoelhorst H, Viti F (2008) Specialist: A dynamic speed limit control algorithm based on shock wave theory. In: IEEE Conference on Intelligent Transportation Systems, Proceedings, ITSC'08. IEEE, Beijing. pp 827-832

19. Hellinga B, Samimi A (2007) Safety evaluations using a real-time crash potential model: Sensitivity to model calibration. In: Proceedings of the ITE Canadian District Annual Conference. ITE Canada, Toronto

20. International Transport Forum (2010) Transport Greenhouse gas emissions, Country data. Research Report. International Transport Forum/OECD, Paris. https://www.itf-oecd.org/sites/default/files/docs/ 10ghgcountry.pdf

21. International Transport Forum (2013) Global Transport Trends in Perspective Statistics Breif: Trends in the transport sector. International Transport Forum/OECD, Paris. https://www.itf-oecd.org/sites/default/ files/docs/2013-12-trends-perspective.pdf

22. Jin HY, Jin WL (2015) Control of a lane-drop bottleneck through variable speed limits. Transp Res C Emerg Technol 58:568-584

23. Khondaker B, Kattan L (2015) Variable speed limit: an overview. Transp Lett 7(5):264-278

24. Krajzewicz D (2011) Traffic simulation with sumo - simulation of urban mobility. In: Barceló J (ed). Fundamentals of traffic simulation, International series in operations research \& management science, vol. 145, chap. 8. Springer, New York and Heidelberg. pp 269-293

25. Krajzewicz D, Erdmann J, Behrisch M, Bieker L (2012) Recent development and applications of SUMO - Simulation of Urban MObility. Int J Adv Syst Meas 5(3\&4):128-138 
26. Krauß S (1998) Microscopic modeling of traffic flow: Investigation of collision free vehicle dynamics. PhD Thesis, Köln

27. Laval JA, Daganzo CF (2006) Lane-changing in traffic streams. Transp Res B Methodol 40:251-264

28. Lee C, Hellinga B, Saccomanno F (2003) Real-time crash prediction model for application to crash prevention in freeway traffic. Transp Res Rec: J Transp Res Board 1840(03-2749):67-77

29. Lee C, Hellinga B, Saccomanno F (2004) Assessing safety benefits of variable speed limits. Transp Res Rec: J Transp Res Board 1897:183-190

30. Lee C, Hellinga B, Saccomanno F (2006) Evaluation of variable speed limits to improve traffic safety. Transp Res C: Emerg Technol 14(3):213-228

31. Li D, Ranjitkar P (2015) A fuzzy logic-based variable speed limit controller. J Adv Transp 49(8):913-927

32. Li D, Ranjitkar P, Ceder A (2014) A logic tree based algorithm for variable speed limit controllers to manage recurrently congested bottlenecks. In: Transportation Research Board $93^{\text {rd }}$ Annual Meeting, Compendium of papers. Transportation Research Board, Washington, D.C

33. Li Z, Liu P, Wang W, Xu C (2014) Development of a control strategy of variable speed limits to reduce rear-end collision risks near freeway recurrent bottlenecks. IEEE Trans Intell Transp Syst 15(2):866-877

34. Liang XR, Wang DQ (2012) Design and simulation of speed limit controller based on fuzzy logic inference. Appl Mech Mater 220-223:988-991

35. Lind G, Strömgren P (2011) Säkerhetseffekter av trafikledning och ITS, Litteraturinventering, v 0.9. Movea, Stockholm

36. Lu XY, Shladover S (2014) Review of variable speed limits and advisories: Theory, algorithms, and practice. Transp Res Rec J Transp Res Board 2423:15-23

37. Maunsell F, Parkman M (2007) M25, Control motorway, Summary report. Technical Report. Highway Agency, Bristol

38. Müller ER, Carlson RC, Kraus W, Papageorgiou M (2015) Microsimulation analysis of practical aspects of traffic control with variable speed limits. IEEE Trans Intell Transp Syst 16(1):512-523

39. Müller ER, Carlson RC, Kraus Jr W, Papageorgiou M (2013) Microscopic simulation analysis of mainstream traffic flow control with variable speed limits. In: IEEE Conference on Intelligent Transportation Systems, Proceedings, ITSC'13. IEEE, Hague

40. Muralidharan A, Horowitz R (2015) Computationally efficient model predictive control of freeway networks. Transp Res C Emerg Technol 58:532-553

41. Nissan A, Bang KL (2006) Evaluation of impacts of the motorway control system (MCs) in stockholm. In: European transport conference -cd-rom edition-, European transport conference; ETC proceedings. Association for European Transport, Strasbourg. p 234

42. Nissan A, Koutsopoulos HN (2011) Evaluation of the impact of advisory variable speed limits on motorway capacity and level of service. Procedia - Soc Behav Sci 16:100-109

43. Samimi A, Hellinga B (2012) Sensitivity of a real-time freeway crash prediction model to calibration optimality. Eur Transp Res Rev 4(3):167-174

44. Schelling I, Hegyi A, Hoogendoorn SP (2011) Specialist-rm - integrated variable speed limit control and ramp metering based on shock wave theory. In: IEEE Conference on Intelligent Transportation Systems, Proceedings, ITSC'11. IEEE, Washington, D.C

45. Scora G, Barth M (2006) Comprehensive Modal Emission Model (CMEM), version 3.01, User's guide. Technical report. University of California, Riverside, Center for Environmental Research and Technology, California

46. Soriguera F, MartÃnez I, Sala M, MenÃ@ndez M (2017) Effects of low speed limits on freeway traffic flow. Transp Res C Emerg Technol 77:257-274

47. Soriguera F, Torné JM, Rosas D (2013) Assessment of dynamic speed limit management on metropolitan freeways. J Intell Transp Syst 17(1):78-90

48. Srivastava A, Geroliminis N (2013) Empirical observations of capacity drop in freeway merges with ramp control and integration in a first-order model. Transp Res C Emerg Technol 30:161-177

49. Tampère $C$, Immers L (2007) An extended kalman filter application for traffic state estimation using CTm with implicit mode switching and dynamic parameters. In: IEEE Conference on Intelligent Transportation Systems, Proceedings, ITSC'07. IEEE, Begium. pp 209-216

50. van den, Hoogen E, Smulders S (1994) Control by variable speed signs. results of the dutch experiment. In: $7^{\text {th }}$ International Conference on Road Transport Information and Control. IET, London. pp 145-149
51. van Rossum G (1995) Python tutorial, Technical Report CS-R9526. Technical Report. Centrum voor Wiskunde en Informatica (CWI), Amsterdam

52. van Toorenburg JAC, de Kok ML (1999) Automatic incident detection in the motorway control system MTM. Bureau Transpute, Gouda

53. Varedian M (2013) Hastighetsundersökningen 2012, Resultatrapport. Tech. Rep. 2013:002. Trafikverket, Sweden

54. Wang Y, Papageorgiou M (2005) Real-time freeway traffic state estimation based on extended Kalman filter: a general approach. Transp Res B Methodol 39(2):141-167

55. Wang Y, Papageorgiou M, Messmer A, Coppola P, Tzimitsi A, Nuzzolo A (2009) An adaptive freeway traffic state estimator. Automatica 45:10-24

56. Yu R, Abdel-Aty M (2014) An optimal variable speed limits system to ameliorate traffic safety risk. Transp Res C Emerg Technol 46:235-246

57. Zegeye S, De Schutter B, Hellendoorn J, Breunesse E (2011) Reduction of area-wide emissions using an efficient model-based traffic control strategy. In: IEEE Forum on Integrated and Sustainable Transportation Systems, FISTS. IEEE, Vienna. pp 239-244

58. Zegeye SK, De Schutter B, Hellendoorn H, Breunesse E (2009) Reduction of travel times and traffic emissions using model predictive control. In: IEEE American Control Conference ACC'09. IEEE, St. Louis

59. Zhang L, Levinson D (2004) Some properties of flows at freeway bottlenecks. Transp Res Rec J Transp Res Board 1883:122-131

\section{Submit your manuscript to a SpringerOpen ${ }^{\circ}$ journal and benefit from:}

- Convenient online submission

- Rigorous peer review

Open access: articles freely available online

- High visibility within the field

- Retaining the copyright to your article

Submit your next manuscript at $>$ springeropen.com 